\title{
Atitudes e conhecimentos em relação à velhice em estudantes de graduação em educação e em saúde: subsídios ao planejamento curricular
}

\author{
Attitudes and beliefs toward aging among \\ undergraduate students from the education and \\ health fields: curriculum planning contributions
}

\author{
Anita Liberalesso NERI \\ Mariana Dias JORGE²
}

\begin{abstract}
Resumo
Esta pesquisa descreve e compara atitudes e conhecimentos sobre velhice em alunos de graduação, com uma amostragem de 277 alunos de Pedagogia, Educação Física, Medicina e Enfermagem, de 18 a 43 anos ( $M=23, \mathrm{DP}=3,39$ ), sendo 190 mulheres; $50,0 \%$ freqüentaram disciplina; $60,0 \%$ estudaram tópico sobre velhice; $62,0 \%$ convivem e $32,0 \%$ trabalham com idosos. Os instrumentos utilizados foram a) Diferencial semântico - 30 itens bipolares - domínios agência, cognição, relações sociais e persona; e b) Escala de conhecimentos - 25 itens - físicos, psicológicos e sociológicos. São apresentados os seguintes resultados: a) atitudes positivas, principalmente nos mais jovens, mulheres e que convivem com idosos; b) Baixo nível de acertos em conhecimentos ( $M=41,0 \%, D P=10,3 \%)$; c) Os que estudaram sobre velhice (cursos de Enfermagem, Educação Física e Medicina) sabem mais do que os que não estudaram (Educação); d) Atitudes e conhecimentos positiva e significantemente correlacionados. Portanto, educar e atender adequadamente os idosos dependem da oferta de estruturas de conhecimento e de oportunidades para desenvolver habilidades e valores específicos.
\end{abstract}

Palavras-chave: atitudes em relação à velhice; crenças em relação à velhice; gerontologia; estudantes universitários.

\begin{abstract}
The aim of this paper is describe and compare the undergraduates' attitudes and beliefs toward aging. Two hundred and seventy seven students (190 women) aged from 18 to $43(M=23 ; S D=3,39)$ have participated in this investigation. Fifty percentagem of them had attended to disciplines; $60 \%$ studied aging issues; $62 \%$ had social contacts and $32 \%$ worked with aged people. The instruments were a) Semantic differential: 30 bi-polar items (DSS) reflecting agency, cognition, social relations and social image; b) Knowledge scale: 25 multiple choice items reflecting physical, psychological and sociological knowledge. The according to the results: a) Positive attitudes, mostly among the youngest, women and those who lived close to aged people; b) Aging right answers low rate ( $M=41 \%, S D=10,3 \%) ; c)$ Those that have studied aging matters (Nursing, Physical Education and Medicine students) knew more than those who did not (Education); d) Attitudes and knowledge were positively and significantly correlated. It was concluded that aging attendance and education depends on the offering of opportunities and knowledge structure.
\end{abstract}

Key words: aged, attitudes toward aging; beliefs toward aging; gerontology; undergraduate students.

\section{v V V}

1 Professora Doutora, Departamento de Psicologia Educacional, Faculdade de Educação, Universidade Estadual de Campinas. Av. Bertrand Russell, 801, Cidade Universitária, 13085-970, Campinas, SP, Brasil. Correspondência para/Correspondence to: A.L. NERI. E-mail: <anitalbn@uolo.com.br>.

2 Acadêmica, Curso de Pedagogia, Faculdade de Educação, Universidade Estadual de Campinas. Campinas, SP, Brasil. Bolsista PIBIC/CNPq, $2003-2004$. 
A noção de atitude como predisposição socialmente aprendida para o comportamento é aceita clássica e consensualmente pelos psicólogos sociais. Da mesma forma, aceita-se que as atitudes se organizam em sistemas cujo caráter distintivo é o avaliativo, que se expressa afetivamente em termos de intensidade (mais x menos, ou maior x menor) e direção (positiva $x$ negativa); que orientam a ação e que têm um componente cognitivo (Osgood, Suci \& Tannembaum, 1957). O componente cognitivo é uma estrutura de conhecimentos ou de crenças compartilhadas com outras pessoas. Tais estruturas possibilitam ao indivíduo organizar e hierarquizar as informações recebidas e assim auxiliam a construção de suas noções sobre o mundo externo e sobre si mesmo. São compartilhadas com o grupo no qual ele está inserido, que por sua vez mantém estreita ligação com a organização, com a cultura e com o grau de envolvimento e a participação dos seus integrantes. Por conseguinte, o que é tido como uma crença válida para determinado grupo pode não ser em outro, dependendo dos eventos sociais, culturais, históricos e políticos que afetam e afetaram a experiência individual e coletiva. Atitudes e crenças são, assim, assumidas como eventos reguladores do comportamento de indivíduos e grupos. Ambos os conceitos têm largo trânsito quando o interesse é conhecer preditores afetivos e cognitivos do comportamento em relação a objetos sociais, caso da velhice e dos idosos.

A velhice é um conceito historicamente construído que se inscreve na dinâmica das atitudes, das crenças e dos valores da sociedade. A marca social da velhice é estar em oposição à juventude, motivo pelo qual é recorrente a oscilação entre a idealização e a depreciação do idoso. Os estereótipos - que são crenças generalizadas sobre os atributos ou características que definem um determinado grupo social, como, por exemplo, o dos idosos - são transmitidos pela educação

$\geq \quad$ e associam-se a práticas sociais discriminativas (Neri, 觉 Cachioni \& Resende, 2002).

Butler (1969) definiu o fenômeno do preconceito em relação aos idosos como uma forma de intolerância comparável à racial, à religiosa ou àquela que se estabelece com base no sexo das pessoas, dando origem a políticas e práticas discriminativas. Por exemplo, no Brasil, observa-se uma sutil campanha de atribuição dos déficits da previdência e do sistema de saúde ao aumento da população idosa, na qual se baseia a negação de reajustes das aposentadorias e de recursos terapêuticos aos mais velhos (Neri, 2003). No final dos anos 1970, Kalish denunciou a existência de estereótipos compassivos, focalizados no velho dependente, incapaz e sem poder político, os quais podem acirrar a intolerância dos mais jovens, principalmente em épocas de desemprego e recessão, em que políticas de proteção aos mais velhos e a outras minorias podem parecer estar roubando oportunidades aos mais jovens. No âmbito da família e das instituições, a vitimização dos idosos contribui para que Ihe seja oferecido um tratamento superprotetor que, em vez de ampliar-Ihes as chances de autonomia e independência, transformaos em seres cada vez mais dependentes.

Palmore (1990) acrescentou novo elemento à análise das atitudes em relação à velhice quando estabeleceu os conceitos de preconceito positivo e negativo. Para o autor, considerar todos os idosos como sábios esconde uma falsa crença positiva e um compromisso pretensamente positivo com os velhos que não condiz com a realidade. Preconceitos e estereótipos positivos podem ser disfuncionais para a auto-estima, o senso de auto-eficácia e a inserção social dos idosos. Levy (2001) chamou a atenção para os preconceitos implícitos, que existem e operam sem o conhecimento ou o controle conscientes das pessoas, que assim podem achar normais as formas de tratamento discriminativas a que são submetidos no trabalho, na propaganda ou nos serviços de saúde.

Segundo Schaie (1993), na formação das atitudes e crenças em relação à velhice têm grande peso as opiniões e as ações dos cientistas e dos profissionais de ajuda, as quais muitas vezes são preconceituosas. Vejam-se exemplos: a) considerar os idosos como uma categoria homogênea, sem levar em conta que diferentes condições de saúde e de estilo de vida refletem-se em diferentes manifestações de competência comportamental; b) atribuição prévia de dependência física, depressão e doença aos sujeitos idosos; c) inadequação de instrumentos, instruções, equipamentos e ambientes usados nas situações de avaliação e de coleta de dados; d) confusão entre os efeitos da velhice com os da pobreza, da doença, ou do baixo nível educacional; e) desconsideração das circunstâncias históricas como determinantes de estilos 
de vida e de valores dos mais velhos; f) desconsideração dos limites que o envelhecimento normal impõe ao funcionamento dos seres humanos, em favor da falsa crença no poder irrestrito da ciência de impedi-lo ou de restaurar a juventude.

Em análise da literatura brasileira sobre velhice, entre os anos de 1975 e 1995, Neri (1997) encontrou reflexos das condições acima citadas, além de clara tendência ateórica e de pulverização e descontinuidade de temas e campos de pesquisa. Os traços de preconceito na pesquisa brasileira sobre velhice refletem os preconceitos científicos que tradicionalmente caracterizaram a pesquisa norte-americana e européia, notadamente os advindos da Gerontologia Social e da Medicina.

Muito provavelmente os preconceitos dos pesquisadores, dos profissionais, dos políticos, das instituições sociais, da mídia e das pessoas de modo geral são afetados pelo baixo status dos idosos na sociedade brasileira. Os preconceitos decorrem mais da pobreza, do baixo nível educacional e das doenças que lhes são inerentes do que propriamente de sua condição de serem velhos numa sociedade que supervaloriza a juventude. Ademais, e aqui não há distinção em relação aos dos países ricos, nossos idosos formam uma categoria rejeitada porque a velhice é vista como período de declínio, de dependência e, mais do que isso, como a antecâmara da morte (Neri, 2003). Apesar de tudo, é temerário afirmar que predominam atitudes negativas em relação à velhice, quer na pesquisa, quer nas categorias profissionais, quer nas sociedades ou na sociedade brasileira (Neri, 1991, 1997). Falsos ou escassos conhecimentos gerontológicos dão origem a falsas avaliações sobre a velhice e se traduzem em preconceitos positivos e negativos em relação a ela, caracterizados pela supergeneralização de características desejáveis ou indesejáveis compartilhadas por uma parte dos idosos e não por todos, o que resulta em denominações, afirmações, formas de tratamento, práticas e políticas inapropriadas em relação aos idosos (Cachioni, 2002; Neri, 1977).

À medida que cresce a população idosa no Brasil, aumentam as demandas nas áreas de prestação de serviços, pesquisa e políticas públicas, abrindo-se novos espaços ocupacionais. Do profissional especializado em gerontologia espera-se que responda com competência teórica e prática aos desafios do envelhecimento individual e populacional. Conseqüentemente, é necessário que se invista na formação de recursos humanos. Os profissionais têm um importante papel na realização de pesquisas sobre as características dos idosos, na busca dos determinantes do envelhecer bem e, principalmente, na divulgação dos conhecimentos acerca das ações que previnem o desajustamento, a doença, a incapacidade. As necessidades evolutivas dos idosos requerem um foco não apenas sobre declínio e mudança, como também sobre a manutenção do controle de sua vida (Neri, 2004).

Quando a presença dos idosos na sociedade torna-se mais notada, não somente por causa do seu aumento numérico, mas também porque a melhora relativa do nível de vida de parte deles faz com que tenham mais visibilidade social e que passem a demandar mais por serviços especializados, as profissões e as instituições sociais tendem a começar a desenvolver ou a consolidar formas de atender a essa clientela e a reconhecer que é importante resguardar e investir na boa qualidade de vida na velhice, em favor da saúde econômica da própria sociedade. No Brasil essas mudanças já se fazem notar na promulgação de leis e normas específicas de atendimento aos idosos, tais como o Estatuto do Idoso, em 2003, e a Política Nacional do Idoso, em 1996, e em providências advindas de vários setores da sociedade em relação a cuidar da saúde, promover a educação e a participação social e desenvolver profissões ligadas ao atendimento aos idosos (Lopes, 2001). Nesse contexto a pesquisa sobre educação gerontológica e, dentro dela, a investigação de atitudes e crenças de alunos universitários são de grande interesse.

Este estudo nasceu da preocupação com a responsabilidade da universidade pela formação de recursos humanos qualificados para atender a velhice e o envelhecimento de indivíduos e populações em suas várias manifestações normativas, ótimas e patológicas.

Pretende-se contribuir para aumentar a base de conhecimentos sobre educação gerontológica, investigando e discutindo dados relativos às atitudes e às crenças de alunos de graduação das áreas da saúde e da educação de uma grande universidade pública, pois, no futuro, eles trabalharão em áreas cruciais ao bem-estar da população e ao progresso social. Além disso, conhecer esses mediadores do comportamento ajuda a compreender as práticas sociais e acadêmicas e 
a propor alternativas educacionais de médio e longo prazo para elas.

Foram definidos como objetivos para esta pesquisa: a) descrever a intensidade, a direção e o conteúdo das atitudes de alunos de cursos de graduação de Medicina, Educação Física, Enfermagem e Pedagogia em relação aos idosos; b) identificar os conhecimentos básicos dos alunos sobre os aspectos físicos, sociais e psicológicos da velhice; c) caracterizar relações univariadas, bivariadas e multivariadas entre atitudes e conhecimentos dos alunos em relação à velhice, e de cada um desses construtos com as variáveis gênero, idade, curso de origem, experiências acadêmicas e de trabalho e convivência com idosos; d) analisar a consistência interna das duas escalas na amostra.

\section{Método}

\section{Participantes}

Foram 277 estudantes, sendo 111 do curso de Pedagogia, 98 do curso de Educação Física, 39 da Medicina e 29 da Enfermagem ${ }^{3}$. Dentre eles, 190 eram mulheres, que predominaram nos cursos de Pedagogia e de Enfermagem. Oitenta e sete $(31,4 \%)$ eram homens, distribuídos principalmente pelos cursos de Medicina e Educação Física. A comparação dessas freqüências pelo teste $\chi^{2}$ mostrou que as diferenças eram estatisticamente significantes $\left(\chi^{2}=74,39 ; G L=3\right.$ e $\left.p<0,0001\right)$. A idade dos sujeitos variava entre 18 e 43 anos, com média de 23 anos e DP de 3,39; 77,7\% tinham entre 20 e 24 anos. A maioria (78,7\%) estava no 40, $5^{\circ}$ ou 60, 15,4\% no $7 \circ, 8^{\circ}$ ou $9 \circ, 4,4 \%$ no $1^{\circ}, 2^{\circ}$ ou $3^{\circ}$, e $1,5 \%$ no $10^{\circ}, 11^{\circ}$ ou $12^{\circ}$ semestres.

\section{Procedimento}

Os alunos formaram amostra de conveniência. Foi voluntária sua participação numa única sessão coletiva de coleta de dados (duração média de 30 minutos), mediante formulário impresso e auto-instrucional. Foram respeitadas as exigências éticas para pesquisa com seres humanos e assinado o termo de consentimento informado, que esclarecia os objetivos, o direito a sigilo, o caráter optativo da participação e o direito de abandonar a pesquisa a qualquer momento. O projeto foi aprovado pelo Comitê de Ética em Pesquisa da Faculdade de Ciências Médicas, Universidade Estadual de Campinas.

\section{Instrumentos}

Fizeram parte do formulário da pesquisa um questionário levantando dados sociodemográficos e acadêmicos (idade, gênero, curso e série), uma questão dicotômica avaliando a convivência dos alunos com idosos (Você convive regularmente com seus avós ou com outros parentes idosos?), uma outra levantando se tinham experiência de trabalho com essa população (Você desenvolve ou já desenvolveu algum trabalho profissional ou voluntário envolvendo algum tipo de atendimento a idosos?), e três sobre suas experiências acadêmicas com o tema velhice (Você já cursou alguma disciplina sobre velhice? Em alguma das disciplinas quejá freqüentou, você teve algum tópico sobre velhice? Você já leu algum livro sobrevelhice?).

Para avaliar as atitudes em relação à velhice foi utilizada uma escala diferencial semântica (Neri, 1991, 1995) e validada em várias pesquisas (Cachioni, 1998; Cachioni, 2002; Pereira da Silva, 1999; Resende, 2001). A estrutura da escala é descrita em termos cognitivos ou relativos à capacidade de processamento da informação e de solução de problemas, com reflexos sobre a adaptação social (dez itens); de agência, isto é, autonomia e instrumentalidade para realização (seis itens); de relacionamento interpessoal, cobrindo aspectos afetivo-motivacionais, que se refletem na interação social (sete itens); de persona (sete itens), refletindo rótulos sociais comumente usados para designar ou discriminar pessoas idosas. Os itens escolhidos para figurar na escala são correntes na literatura de pesquisa sobre atitudes em relação à velhice e na literatura sobre o desenvolvimento ao longo do curso de vida.

Para avaliar os conhecimentos básicos sobre velhice foi usada a Escala Palmore-Neri-Cachioni, uma versão do Palmore Aging Quiz (Harris, Changas \& Palmore, 1996; Palmore, 1977). Continha 25 itens de

$\boldsymbol{\nabla} \boldsymbol{\nabla} \boldsymbol{\nabla} \boldsymbol{\nabla}$

3 O projeto previa a participação de 400 alunos, sendo 100 de cada um dos cursos, metade homens e metade mulheres, mas impedimentos vários ligados 130 à vida acadêmica dos institutos e faculdades inviabilizaram o acesso a esse total de casos. 
múltipla escolha cobrindo os domínios físico, cognitivo, psicológico e social, os quais foram submetidos à validação de conteúdo e de consistência interna em amostra de 102 professores universitários dedicados à educação gerontológica, tendo alcançado um índice $\alpha \leq 0,80$ (Cachioni, 2002).

\section{Resultados}

Os dados foram submetidos à análise descritiva mediante testes estatísticos paramétricos e não-paramétricos. Os alunos foram agrupados por curso, gênero e idade e segundo a sua experiência acadêmica, de convivência e de trabalho com idosos e foram comparados quanto aos escores obtidos na avaliação de conhecimentos e de atitudes em relação à velhice. Foram feitas análises de variância (ANOVA) quando as comparações envolviam três ou mais grupos e testes " $t$ " de Student quando se tratou da comparação entre as médias de dois grupos. Análises de correlações bivariadas entre os escores da escala de atitudes e os percentuais de acertos na escala de conhecimentos foram feitas a partir do modelo de Spearman. Para a análise multivariada dos mesmos dados foi adotado o método de análise de correspondência múltipla. O nível de significância adotado para todos os testes estatísticos foi de 5\%. A consistência interna das escalas foi calculada segundo o índice de Cronbach.

Quase metade da amostra (49,10\%) havia freqüentado alguma disciplina e $60,00 \%$ dos alunos relataram já terem estudado algum tópico sobre velhice. Os cursos nos quais os alunos foram mais expostos a disciplinas sobre velhice foram Enfermagem (100,00\%) e Educação Física (73,74\%). Os menos expostos foram os de Pedagogia (82,88\%) e Medicina (58,97\%). Os alunos da Enfermagem, da Educação Física e da Medicina foram os mais expostos a tópicos sobre velhice em seus currículos, com, respectivamente, 93,10\%, 78,45\% e $56,41 \%$ de respostas afirmativas. Os de Pedagogia foram os menos expostos (63,06\%). Da amostra, 86,30\% e dos alunos da Pedagogia, 90,99\% disseram nunca ter lido nenhum livro sobre velhice. Disseram conviver com idosos 62,00\%, mas apenas 32,00\% relataram trabalhar com idosos, dentre eles 64,29\% da Enfermagem.

Duzentos e sessenta e dois alunos responderam por completo à Escala Neri de Atitudes em Relação à Velhice (103 da Pedagogia, 93 da Educação Física, 38 da
Medicina e 28 da Enfermagem). Os escores médios indicam uma tendência global positiva, uma vez que as médias e medianas foram todas menores do que 3. Entre os quatro fatores da escala, foi em agência que o grupo pontuou mais baixo. Duzentos e cinqüenta e um alunos responderam completamente à Escala Palmore-NeriCachioni de Conhecimentos em Relação à Velhice (102 da Pedagogia, 86 da Educação Física, 36 da Medicina e 27 da Enfermagem). Os percentuais de acertos foram baixos. O máximo foi de 68,0\% e média de 41,0\% (DS=10,3\%). As questões menos acertadas foram as do domínio social.

Na Figura 1 são apresentados os escores médios do grupo na escala de atitudes. Observe-se que a tendência geral foi positiva, uma vez que somente um terço dos itens ultrapassou o ponto médio da escala (3). As avaliações mais positivas foram para os itens sábio, interessado pelas pessoas e agradável. As mais negativas foram para rápido, valorizado e aceito. Em relações sociais ocorreu a maior proporção de médias mais positivas. Na Figura 2 aparece o número de acerto por item e por domínio na escala de conhecimentos em relação à velhice. $O$ domínio em que a freqüência de acertos foi maior foi o físico. No domínio social foi onde ocorreram menos acertos.

As Tabelas 1 e 2 apresentam as estatísticas das ANOVA e dos testes " $t$ " em que ocorreram diferenças estatisticamente significantes quanto ao desempenho dos grupos nas duas escalas. Foram conduzidos testes post-hoc de Tukey para avaliar, em cada caso, qual dos grupos-comparação se diferenciou.

O cálculo das correlações de postos entre os escores médios nas duas escalas e seus domínios evidenciou correlações significativas embora fracas entre a idade e o escore total, e entre a idade e os escores nos domínios persona, agência e social (escala de atitudes); entre os acertos totais na escala de conhecimentos e o escore total e por domínios da escala de atitudes; entre o percentual de acertos no domínio cognitivo da escala de conhecimentos e o escore total e os dos domínios cognição, agência e social da escala de atitudes; entre o percentual de acertos no domínio físico da escala de conhecimentos e o escore total e nos domínios cognição e agência da escala de atitudes; entre os percentuais de acertos no domínio psicológico da escala de conhecimentos e o escore médio total e nos domínios cognição e social da escala de atitudes. 


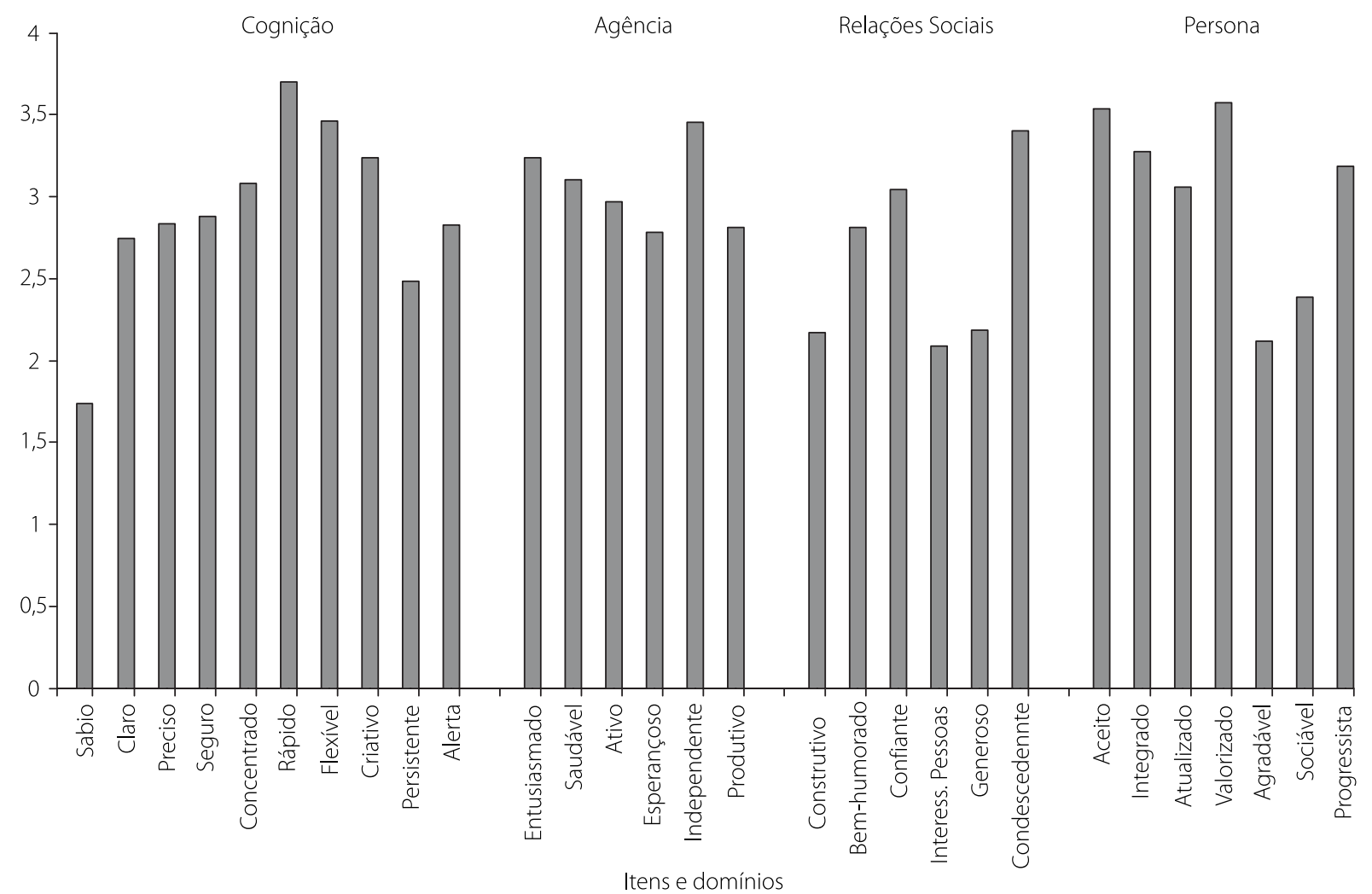

Figura 1. Escores médios nos itens de cada um dos domínios da Escala de Atitudes em Relação à Velhice ( $n=262)$.

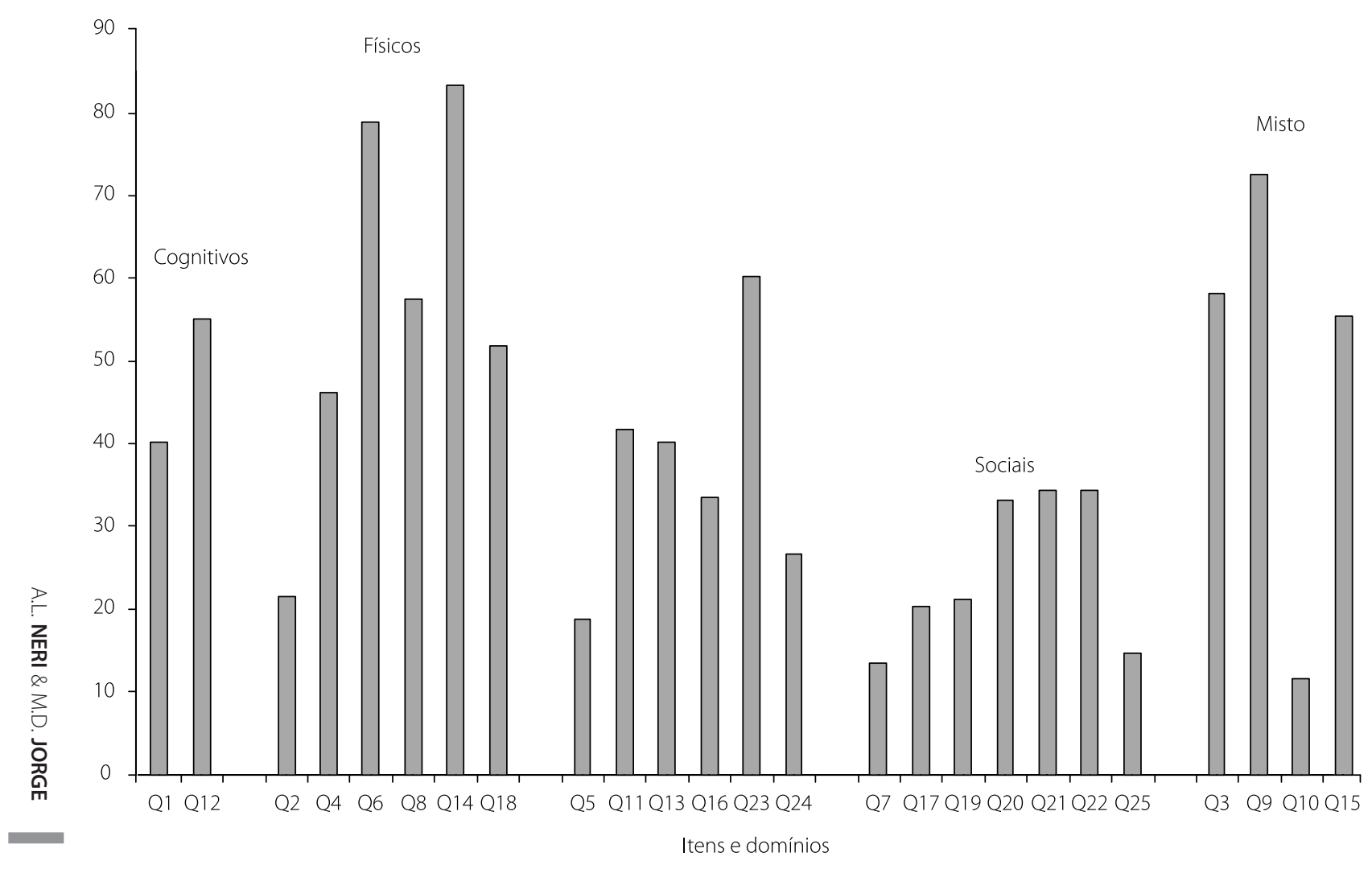

132 Figura 2. Porcentagens de acertos nos itens de cada um dos domínios da Escala de Conhecimentos em Relação à Velhice ( $n=252)$. 
Tabela 1. Estatísticas relativas às comparações entre escores médios da escala de atitudes em relação à velhice que resultaram em diferenças estatisticamente significantes entre os grupos considerados.

\begin{tabular}{lcccccccc}
\hline Variável & Grupos & Foco & Média \pm & DP & Máxima & Mediana & Mínima & $p$-valor \\
\hline Idade & $<22=143$ & Escore total & $\underline{2,74} \pm 0,47$ & 3,67 & 2,80 & 1,23 & 0,0054 \\
& $>22=115$ & na escala & 2,88 & $\pm 0,37$ & 3,70 & 2,90 & 1,80 & \\
& $<22=143$ & Agência & $\underline{2,90} \pm 0,60$ & 4,17 & 3,00 & 1,33 & 0,0121 \\
& $>22=115$ & & 3,08 & $\pm 0,51$ & 4,67 & 3,00 & 1,83 & \\
& $<22=143$ & Relações & $\underline{2,43} \pm 0,51$ & 4,14 & 2,43 & 1,43 & 0,0332 \\
& $>22=115$ & sociais & 2,56 & $\pm 0,46$ & 3,71 & 2,57 & 1,14 & \\
& $<22=143$ & Persona & $\underline{2,86} \pm 0,58$ & 4,14 & 3,00 & 1,00 & 0,0088 \\
& $>22=115$ & & 3,04 & $\pm 0,51$ & 4,00 & 3,14 & 1,71 & \\
Convivência com idosos & Sim $=164$ & Persona & $\underline{2,87} \pm 0,55$ & 4,00 & 3,00 & 1,00 & 0,0121 \\
& Não $=98$ & & 3,05 & $\pm 0,51$ & 4,14 & 3,14 & 1,71 & \\
\hline
\end{tabular}

Tabela 2. Estatísticas relativas às comparações entre escores médios da escala de conhecimentos em relação à velhice que resultaram em diferenças estatisticamente significantes entre os grupos considerados.

\begin{tabular}{|c|c|c|c|c|c|c|c|c|c|}
\hline Variável & Grupos & Foco & Média & \pm & DP & Máxima & Mediana & Mínima & $p$-valor \\
\hline \multirow[t]{4}{*}{ Gênero } & Masc $=78$ & Escore total & $\underline{43,33}$ & \pm & 10,34 & 68,00 & 44,00 & 24,00 & 0,0146 \\
\hline & Fem $=173$ & & 39,91 & \pm & 10,16 & 64,00 & 40,00 & 12,00 & \\
\hline & & Domínio social & $\underline{28,75}$ & \pm & 17,91 & 71,43 & 28,57 & & 0,0079 \\
\hline & & & 22,54 & \pm & 14,29 & 57,14 & 14,29 & & \\
\hline \multirow[t]{2}{*}{ Idade } & $<22=$ & Domínio misto & $\underline{22,46}$ & \pm & 14,94 & 57,14 & 14,29 & 0 & 0,0288 \\
\hline & $>22=$ & & 26,87 & \pm & 16,46 & 71,43 & 28,57 & 0 & \\
\hline \multirow[t]{12}{*}{ Curso } & Ped $=102$ & Escore total & 38,08 & \pm & 10,93 & 68,00 & 36,00 & 12,00 & $0,025^{\text {(a) }}$ \\
\hline & $E n f=7$ & & 43,85 & \pm & 9,25 & 64,00 & 40,00 & 28,00 & \\
\hline & Med=36 & & 43,56 & \pm & 10,15 & 64,00 & 44,00 & 24,00 & \\
\hline & Ed.Fís $=86$ & & 42,42 & \pm & 9,23 & 68,00 & 42,00 & 24,00 & \\
\hline & Ped $=102$ & Domínio físico & 48,69 & \pm & 20,80 & 83,33 & 50,00 & 0,00 & $0,0001^{\text {(b) }}$ \\
\hline & $E n f=27$ & & 67,90 & \pm & 20,63 & 100,00 & 66,67 & 16,67 & \\
\hline & Med=36 & & 60,65 & \pm & 19,17 & 100,00 & 50,00 & 33,33 & \\
\hline & Ed.Fís $=86$ & & 60,47 & \pm & 18,71 & 100,00 & 66,67 & 16,67 & \\
\hline & Ped $=102$ & Domínio social & 24,23 & \pm & 15,15 & 57,14 & 28,57 & 0 & $0,0599^{(c)}$ \\
\hline & $E n f=27$ & & 18,52 & \pm & 13,62 & 42,86 & 14,29 & 0 & \\
\hline & Med=36 & & $\underline{29,37}$ & \pm & 16,00 & 57,14 & 28,57 & 0 & \\
\hline & Ed.Fís=86 & & 24,58 & \pm & 16,49 & 71,43 & 28,57 & 0 & \\
\hline \multirow{2}{*}{$\begin{array}{l}\text { Freqüência à disciplina } \\
\text { sobre velhice }\end{array}$} & $\operatorname{Sim}=119$ & Domínio físico & $\underline{61,34}$ & \pm & 20,35 & 100,00 & 66,67 & 0 & 0,0005 \\
\hline & Não=132 & & 52,15 & \pm & 20,64 & 100,00 & 50,00 & 0 & \\
\hline \multirow{2}{*}{$\begin{array}{l}\text { Estudo tópico sobre } \\
\text { velhice }\end{array}$} & $\operatorname{Sim}=150$ & Domínio físico & $\underline{59,11}$ & \pm & 20,58 & 100,00 & 66,67 & 0 & 0,0145 \\
\hline & Não=100 & & 52,50 & \pm & 21,11 & 100,00 & 50,00 & 0 & \\
\hline \multirow[t]{2}{*}{ Trabalha com idosos } & Sim $=79$ & Domínio físico & $\underline{61,60}$ & \pm & 18,37 & 100,00 & 66,67 & 33,33 & 0,0084 \\
\hline & Não=170 & & 54,12 & \pm & 21,67 & 100,00 & 50,00 & 0,00 & \\
\hline
\end{tabular}

(a) diferenças significativas pelo teste post-hoc de Tukey: Enfermagem $\neq$ Pedagogia; Medicina $\neq$ Pedagogia; (b) diferenças significativas pelo teste post-hoc de Tukey: Enfermagem $\neq$ Pedagogia; Medicina $\neq$ Pedagogia; Educação Física $\neq$ Pedagogia; (c) diferenças significativas pelo teste post-hoc de Tukey: Enfermagem ₹ Medicina; Ped= Pedagogia; Enf= Enfermagem; Med= Medicina; Ed. Fís= Educação Física.

A análise de correspondência múltipla oferece como resultado uma noção sintética dos padrões de variação conjunta das variáveis categóricas, os quais, no caso desta pesquisa, foram: 1) os sujeitos com atitudes mais positivas foram os mais jovens ( $\leq 22$ anos), do gênero feminino, que conviviam com idosos, faziam Medicina e tiveram menor pontuação na escala de conhecimentos; 2) os com atitudes mais negativas eram 
mais velhos ( $\geq 23$ anos), homens, que não conviviam com idosos, faziam Educação Física e pontuaram mais alto na escala de conhecimentos.

Foram calculados os coeficientes de consistência interna para as duas escalas na amostra estudada. A escala de atitudes em relação à velhice alcançou um índice $\alpha=0,891$ (os índices para os domínios foram 0,776 para cognição; 0,716 para agência; 0,607 para relações sociais e 0,732 para persona). A escala de conhecimentos básicos sobre velhice teve baixa consistência $(a=0,488)$, assim como foi baixa a consistência dentro dos seus domínios.

\section{Discussão}

A maior barreira para a transformação de atitudes e de comportamentos em relação à velhice é a falta de conhecimento científico entre os profissionais de educação e de saúde e a falta de esclarecimento de pessoas de todas as idades sobre as características e as potencialidades do envelhecimento (Bringle \& Kremer 1993; Cachioni, 2002; Cachioni \& Neri, 2004a,b; Fajelmelhin, 2004; Hawkins, 1996; Lowenstein, 2001, 2004; McConatha, Hayta, Rieser-Danner \& Polat, 2004; Mehta, Tan \& Joshi, 2002; Meyer, 2003; Prudent \& Tan, 2002; Tan, Zahng \& Fan, 2004).

Os dados desta pesquisa colocaram em evidência o papel importante dos estudos formais no estabelecimento de conhecimentos específicos sobre velhice, uma vez que os alunos que tiveram disciplinas teóricas e práticas (pela ordem os da Enfermagem, os da Educação Física e os da Medicina) mostraram conhecer mais sobre os aspectos físicos, psicológicos e sociais do envelhecimento do que os que não tiveram, caso dos alunos da Educação, nunca antes expostos nem a disciplinas teóricas e nem a estágios com idosos.

Paralelamente, os dados indicam que esses conhecimentos não dependem do senso comum, ou que lidar com idosos não é nem uma atividade que dispensa informação específica e nem uma atividade em que a intuição seja suficiente para garantir o sucesso. A baixa correlação interna entre os itens da escala de conhecimentos pode ser atribuída à desorientação dos alunos, à não compreensão dos itens ou à ambigüidade na redação de algumas alternativas.
Embora fracas, ocorreram correlações significantes entre os escores das escalas de conhecimentos e de atitudes em relação à velhice, mostrando que há uma interação recíproca entre estudar sobre velhice, fazer estágios com idosos e apresentar disposições afetivas favoráveis em relação a eles. É bem provável que essas relações tenham sido mediadas por outra variável de cunho afetivo, qual seja a convivência com idosos fora do âmbito acadêmico, visto que as alunas mais jovens da Medicina, que não tinham conhecimentos formais sobre velhice e que pontuaram mais baixo na escala de conhecimentos, apresentaram atitudes mais positivas do que os alunos mais velhos, homens, da Educação Física e que haviam estudado sobre velhice. Talvez esses, mais do que aquelas, tenham sido expostos a experiências em que as deficiências da velhice no âmbito funcional tenham ficado mais evidentes. Os estudantes que tinham pouca convivência com idosos não teriam tido chance de desenvolver atitudes positivas que pudessem contrabalançar a rejeição oriunda do contato com informações sobre o declínio e a incapacidade da velhice.

Foi verificado que os alunos que relataram conviver com idosos pontuaram mais favoravelmente nos itens relacionados a estereótipos negativos de velhice (os do domínio persona). Esses dados sugerem que a convivência intergeracional seja importante fonte de aprendizagem de atitudes em relação a idosos e que possa predispor favoravelmente as pessoas a estudos formais em relação à velhice. Quanto à pontuação mais positiva de todas ter sido no domínio social, pode ter ocorrido pela interação com experiências afetivas favoráveis ou então como um efeito da expectativa dos respondentes quanto a quais seriam as respostas mais adequadas aos itens de natureza mais claramente afetiva, que, uma vez respondidos negativamente, denotariam atitude preconceituosa. Os itens em que os sujeitos pontuaram menos positivamente foram os de cognição e da agência, o que sugere em parte a influência das informações sobre velhice, em parte um certo realismo decorrente do estudo e da exposição ao trabalho com a clientela.

Quanto mais os programas educacionais puderem colocar os estudantes em contato com os idosos para que tenham experiências reais e pessoais com essa clientela; mostrarem a eles a diversidade 
existente na população idosa e a heterogeneidade das experiências de envelhecimento; forem capazes de lhes apresentar os pontos de convergência entre os processos de envelhecimento e desenvolvimento e os ajudarem a desenvolver um apropriado corpo de conhecimentos, habilidades, atitudes e valores, mais eficazes serão quanto à formação de recursos humanos para lidar com a velhice (Altpeter \& Marshal, 2004; Anderson-Hanley, 1999; Bass \& Ferraro, 2000; Cachioni, 2002; Cachioni \& Neri, 1999, 2003, 2004; Cotter et al., 2004; Harris \& Dollinger, 2001; Lowenstein, 2001; Neri, 2000; von Dras, 2004).

Os conhecimentos comumente expressam-se no âmbito discursivo ou do saber falar sobre dados factuais e de procedimentos. Em relação recíproca com as atitudes, eles medeiam as ações de aproximação ou de esquiva em relação aos objetos sociais. As habilidades expressam-se na probabilidade de que, dada uma situação de vida real, o indivíduo execute uma ação que produza uma conseqüência no ambiente. No caso do idoso, as habilidades profissionais expressam-se, por exemplo, em dar orientação verbal para a ação, oferecer ajuda física, avaliar competências, aconselhar ou provocar alterações no ambiente físico e social visando à melhor adaptação do idoso, dentre outras.

As habilidades são mediadas pelas atitudes $e$ pelos conhecimentos, muito embora a mera presença de declarações verbais avaliativas (atitudes) ou de enunciados de princípios (conhecimentos) por si só não garantam a presença de habilidades instrumentais para mudar o ambiente externo ou a subjetividade de nenhum educando ou paciente. Eis porque a educação meramente verbal, teórica ou livresca é insuficiente para garantir os repertórios de habilidades profissionais em qualquer área. É necessária a exposição a um volume razoável de experiências diretas orientadas por pistas, modelos de desempenho e informações sobre o desempenho para que se produzam os resultados necessários, expressos em eficácia dos desempenhos-alvo.

As atitudes são disposições avaliativas de caráter essencialmente afetivo em relação a um dado objeto. Expressam-se comumente no dizer, mas nem sempre o dizer guarda relação linear com o fazer, o que significa que esse é o domínio de maior complexidade para a educação, pois se por um lado as atitudes medeiam e influenciam as ações, por outro elas podem servir para escamotear, mascarar ou subverter o curso da ação mais funcional para assegurar o bem-estar e o desenvolvimento de outra pessoa, muito embora o indivíduo possa continuar reafirmando suas avaliações positivas ou ideologicamente corretas (Pavarini \& Neri, 2000).

A não adoção de providências educacionais que compreendam atenção ao desenvolvimento e ao aprimoramento de conhecimentos, habilidades e atitudes pode acarretar menos sucesso no trato com a clientela idosa. Expor os idosos a profissionais inexperientes e pouco qualificados - como notam McCormack e Ford (1999), uma ocorrência muito mais comum do que seria desejável - denota desconhecimento ou ignorância das necessidades dos idosos. Desconhecimento das necessidades dos idosos, por sua vez, denota preconceito em relação a eles como indivíduos e como grupo, preconceito esse comumente associado à avaliação de que não são dignos de merecer atenção e tratamento, dado o fato de serem socialmente dependentes, e de que não compensa investir neles porque estão declinando de forma irreversível em termos físicos e mentais.

Esses julgamentos são parciais, visto que a dependência e o declínio comportam muita variabilidade e visto que existe razoável capacidade de reserva para o desenvolvimento, principalmente na velhice inicial. A adoção de julgamentos que não se apóiam completamente em dados objetivos conduz a práticas sociais discriminativas e freqüentemente injustas. Essa é uma situação que pode assumir contornos mais graves no Brasil, país onde a pobreza, a doença e o baixo nível educacional que atingem um grande contingente de idosos contribuem também para que eles sejam uma população silenciosa e conformista, por isso mesmo mais a mercê de profissionais despreparados.

Pensar a formação de profissionais para atender necessidades educacionais, sociais, físicas e afetivas dos idosos idealmente inclui a consideração de complexas questões macroestruturais e culturais, de que fazem parte as atitudes e os conhecimentos sobre velhice. Faz sentido refletir nas seguintes proposições de Douglass (1998) quanto à formação do profissional que vai atender idosos, tomando como ponto de referência os aspectos 
mencionados da realidade nacional. Segundo esse ponto de vista, o profissional deve: a) conhecer a diversidade e a heterogeneidade do processo de envelhecimento; b) estar envolvido em pesquisas e conhecer os avanços tecnológicos da área; c) reconhecer, tanto na teoria como na prática, a importância da ação interdisciplinar; d) contribuir para implantação de programas de educação para o envelhecimento; e) assumir os desafios da área na promoção da saúde, do bem-estar e da qualidade de vida do idoso; f) deve promover junto à sociedade, por meio da ação educativa, mudanças de percepções e atitudes sobre a velhice e o envelhecimento.

Mudanças paradigmáticas em relação à Educação e à Gerontologia precisarão acontecer para que os conteúdos sobre velhice passem a fazer parte dos currículos de formação de professores e dos de profissionais de ajuda de forma mais sistemática do que hoje. Enquanto a Educação for vista como um processo quase artístico, que se dá apenas nas fases iniciais do desenvolvimento e por meio da atuação da família e da escola, e não como um processo científicotecnológico, cultural e pessoal complexo, que inclui aspectos formais, informais, não formais e intrínsecos ao indivíduo, e que percorre todo o curso de vida, não haverá lugar para a consideração da necessidade de ensinar Gerontologia e de praticar educação gerontológica junto a todas as faixas etárias.

Enquanto o estudo da velhice for confundido com a consideração de aspectos patológicos, e a intervenção junto a idosos for vista apenas como um processo de reabilitação, não haverá lugar para a velhice nos currículos de graduação da área de Ciências Humanas, entre eles os da Educação. Continuarão a predominar preconceitos baseados no senso comum sobre velhice e sobre educação gerontológica, os quais trarão pouco beneficio à melhoria da sociedade e de suas instituições, de seus grupos sociais e de seus indivíduos. Simultaneamente, se os currículos da área da saúde derem atenção predominante a aspectos patológicos e à superespecialização, haverá pouco lugar para a consideração da velhice como uma fase do ciclo de vida e do idoso como indivíduo que, além de doenças, tem uma história, uma identidade e um lugar social inseparável no processo de saúde e doença.

\section{Referências}

Altpeter, M., \& Mashall, V. W. (2003). Making aging real for undergraduates. Educational Gerontology, 29 (9), 739-756.

Anderson-Hanley, C. (1999). Experiential activities for teaching psychology of aging. Educational Gerontology, 25 (5), 449-456.

Bass, S. A., \& Ferraro, K. (2000). Gerontology education in transition: considering disciplinary and paradigmatic evolution. Gerontologist, 40 (1), 97-106.

Bringle, R. G., \& Kremer, J. F. (1993). Evalution of an intergenerational service-learning project for undergraduates. Educational Gerontology, 19 (5), 407-416.

Butler, R. N. (1969). Age-ism: another form of bigotry. The Gerontologist, 9 (2), 243-246.

Cachioni, M. (1999). Envelhecimento bem sucedido e participação numa Universidade para a Terceira Idade: a experiência dos alunos da Universidade São Francisco. Dissertação de mestrado não-publicada em Educação, Universidade Estadual de Campinas.

Cachioni, M. (2002). Quem educa os idosos? Um estudo sobre professores de universidades da terceira idade. Campinas: Átomo Alínea.

Cachioni, M., \& Neri, A. L. (1999). Velhice bem sucedida e educação. In A. L. Neri \& G. G. Debert (Orgs.), Velhice e sociedade (pp.113-140). Campinas: Papirus.

Cachioni, M., \& Neri, A. L. (2004a). Educação e velhice bem-sucedida no contexto das Universidades da Terceira Idade. In A. L. Neri, M. S. Yassuda \& M. Cachioni (Orgs.), Velhice bem sucedida. Aspectos afetivos e cognitivos (pp. 46-69). Campinas: Papirus.

Cachioni, M., \& Neri, A. L. (2004b). Educação gerontológica: desafios e oportunidades. Vivencer: Revista Interdisciplinar sobre o Envelhecimento, 1 (1), 69-78.

Cotter, J. J., Coogle, C. L., Parham, I. A., Head, C., Fulton, L., Watson, K., \& Curtis, A. (2004). Designing a multidisciplinary geriatrics health professsional mentoring program. Educational Gerontology, 30 (2), 107-117.

Douglass, B. E. (1998). The role of private foundations and training in Gerontology. Washington: Association for Gerontology in Higher Education. Technical Report.

Fajelmilehin, B. R. (2004). Attitudes of students in health professions toward caring of older people: needed curricula revisions in Nigeria. Educational Gerontology, 30 (3), 383-390.

Harris, K. D., \& Changas, S. P. (1994). Revision of Palmore's second facts on aging quiz from a true-false to a multiple-choice format. Educational Gerontology, 20 (8), 741-754.

Harris, K. D., Changas, S. P., \& Palmore, B. E. (1996). Palmore's first facts on aging quiz in a multiple-choice format. Educational Gerontology, 22 (6), 575-589.

Harris, L. A., \& Dollinger, S. (2001). Participation in a course on aging: Knowledgem attitude and anxiety about aging 
in oneself and others. Educational Gerontology, 27 (8), 667-668.

Hawkins, M. J. (1996). College student's attitudes toward elderly persons. Educational Gerontology, 22 (3), 271-279.

Kalish, R. (1979). The new ageism and the failures models: a polemic. Gerontologist, 19 (3), 398-402.

Levy, B. R. (2001). Erradication of ageism requires addressing the enemy within. Gerontologist, 41 (5), 578-579.

Lopes, A. (2001). Os desafios da Gerontologia no Brasil. Campinas: Átomo Alínea.

Lowenstein, A. (2001). The multidimensionality of gerontological education: the experience of Israel. Educational Gerontology, 27 (6), 493-506.

Lowenstein, A. (2004). Gerontology coming of age: The transformation of social gerontology into distinct academic discipline. Educational Gerontology, 30 (2), 129-141.

McConatha, J. T., Hayta, V., Rieser-Danner, L., \& Polat, T. S. (2004). Turkish and U.S. attitudes toward aging. Educational Gerontology, 30 (3), 169-183.

Mehta, K. K., Tan, P. P., \& Joshi, V. D. (2000). Singapore social work students: attitudes toward older adults. Asia Pacific Journal of Social Work, 10 (2), 40-54.

Meyer, M. (2003). The current state and developments in Gerontology in European Higher Education. Educational Gerontology, 29 (1), 55-69.

Neri, A. L. (1991). Envelhecer num país de jovens. Significados develho evelhice segundo brasileiros não idosos. Campinas: Unicamp.

Neri, A. L. (1995). Atitudes e crenças em relação à velhice. O que pensa o pessoal do SENAC - São Paulo. Relatório técnico. São Paulo: Senac.

Neri, A. L. (1997). Análise de conteúdo de amostra de dissertações e teses em Psicologia e Ciências Sociais produzidas no Brasil no período 1975-1996. Texto e Contexto: Revista de Enfermagem, 6 (2), 69-105.

Neri, A. L. (2000). A formação de recursos humanos em gerontologia: o papel da pós-graduação. Arquivos Brasileiros de Geriatria e Gerontologia, 4 (3), 99-104.

Neri, A. L. (2003). Atitudes e crenças sobre velhice: análise de conteúdo de textos do jornal O Estado de São Paulo publicados entre 1995 e 2002. In O. R. M. von Simson, A. L. Neri, \& M. Cachioni (Orgs.), As múltiplas faces da velhice no Brasil (pp.13-54). Campinas: Átomo Alínea.

Neri, A. L. (2004). O que a Psicologia tem a oferecer ao estudo e à intervenção no campo do envelhecimento no Brasil, hoje? In A. L. Neri, M. S. Yassuda \& M. Cachioni
(Orgs.), Velhice bem sucedida. Aspectos afetivos e cognitivos (pp.6-23). Campinas: Papirus.

Neri, A. L., Cachioni, M., \& Resende, M. C. (2002). Atitudes em relação à velhice. In E. V. Freitas, L. Py, A. L. Néri, F. A . X. Cançado, M. L. Gorzoni \& S. M. Rocha (Orgs.), Tratado de geriatria e gerontologia (pp.972-980). Rio de Janeiro: Guanabara Koogan.

Neri, A . L., \& Freire, S. A. (2000). Qual a idade da velhice? In A. L. Neri \& S. A. Freire (Orgs.), E por falar em boa velhice (pp.7-19). Campinas: Papirus.

Nogueira, E. J., \& Neri, A. L. (1994). A velhice em textos de literatura infantil brasileira. Proposições, 5 (1), 45-60.

Osgood, C. E., Suci, G. J., \& Tannenbaum, P. H. (1957). The measurement of meaning. New York: Appleton.

Palmore, E. (1977). Facts on aging: a short quizz. Gerontologist, 17 (3), 315-320.

Palmore, E. (1990). Ageism: positive and negative. New York: Springer.

Pavarini, S. C. I., \& Neri, A. L. (2000). Compreendendo autonomia, dependência e independência. Conceitos, atitudes e comportamentos. In M. J. D'Elboux Diogo \& M. A. O. Duarte (Orgs.), Atendimento domiciliar. Umenfoque gerontológico (pp.49-70). São Paulo: Atheneu.

Pereira da Silva, F. (1999). Crenças em relação à velhice, bem-estar subjetivo e motivos para freqüentar Universidade da Terceira Idade. Campinas: Dissertação de mestrado não-publicada, Faculdade de Enfermagem, Universidade Estadual de Campinas.

Prudent, E. S., \& Tan, P. P. (2002). Caribbean students'attitudes toward older adults. Educational Gerontology, 28 (8), 669-680.

Resende, M. C. (2001). Atitudes em relação ao idoso, à velhice pessoale a pessoas portadoras de deficiênciafísica em adultos portadores de deficiência física. Campinas: Dissertação de mestrado não-publicada, Faculdade de Enfermagem, Universidade Estadual de Campinas.

Schaie, K. W. (1993). Ageist language in psychological research. American Psychologist, 48 (1), 49-51.

Tan, P. P., Zhang, N., \& Fan, L. (2004). Student's attitudes toward the elderly in the Pople's Republic of China. Educational Gerontology, 30 (4), 301-314.

Von Dras, D. D., \& Lor-Vang, M. N. (2004). Using an Internet activity to enhance students' awareness of age bias in social perceptions. Educational Gerontology, 30 (4), 261-273.

Recebido em: 8/11/2005

Versão final reapresentada em: 14/12/2005

Aprovado em: 14/12/2005 
